\title{
Right ventricular pressure-volume loop produced with simultaneous application of three-dimensional echocardiography and high-fidelity micromanometry in a patient with pulmonary arterial hypertension
}

Toshitaka Nakaya ${ }^{1}$, Ichizo Tsujino ${ }^{1}$, Junichi Nakamura ${ }^{1}$, Yasuyuki Chiba ${ }^{1}$, and Hiroyuki Iwano $^{1}$

${ }^{1}$ Hokkaido University Graduate School of Medicine

February 19, 2021

\begin{abstract}
Accurate assessment of right ventricular (RV) function is drawing a growing attention. Pressure-volume (PV) loop analysis is the gold standard method for evaluating RV function; however, it is not widely employed due to its invasive nature and complexity. The present report is the first to have drawn a RV PV loop in a patient with pulmonary hypertension, with a simultaneous recording of RV pressure and volume using high fidelity micromanometry and 3D echocardiography. This allows for less invasive and simple assessment of RV function, potentially promoting better understanding and management of pulmonary hypertension and other cardiovascular diseases.
\end{abstract}

Right ventricular pressure-volume loop produced with simultaneous application of threedimensional echocardiography and high-fidelity micromanometry in a patient with pulmonary arterial hypertension

Running head: Right ventricular PV loop produced with 3D echo

\section{Authors:}

Toshitaka Nakaya, MD, $\mathrm{PhD}^{1}$; Ichizo Tsujino, $\mathrm{MD}, \mathrm{PhD}^{1}$, Junichi Nakamura, $\mathrm{MD}^{1}$, Yasuyuki Chiba, $\mathrm{MD}^{2}$, Hiroyuki Iwano ${ }^{2}$

${ }^{1}$ Department of Respiratory Medicine, Hokkaido University Graduate School of Medicine

${ }^{2}$ Department of Cardiovascular Medicine, Hokkaido University Graduate School of Medicine

Disclosures: Ichizo Tsujino is affiliated to an endowed department sponsored by Nippon Shinyaku Co., Ltd., Nippon Boehringer Ingelheim Co., Ltd., and Mochida Pharmaceutical Co., Ltd.

IRB approval: The content of the present report is approved by the Institutional Review Board of Hokkaido University Hospital for Clinical Research (No. 016-0461).

\section{Corresponding author:}

Ichizo Tsujino

Department of Respiratory Medicine, Hokkaido University Graduate School of Medicine, N14, W5, Sapporo, Japan. Tel.: 8111706 5911, Fax: 8111706 7899, Email: itsujino@med.hokudai.ac.jp

Funding:

None 


\title{
Keywords:
}

Pulmonary hypertension, Three-dimensional echocardiography, Right ventricular function

\begin{abstract}
Accurate assessment of right ventricular (RV) function is drawing a growing attention. Pressure-volume (PV) loop analysis is the gold standard method for evaluating RV function; however, it is not widely employed due to its invasive nature and complexity. The present report is the first to have drawn a RV PV loop in a patient with pulmonary hypertension, with a simultaneous recording of RV pressure and volume using high fidelity micromanometry and 3D echocardiography. This allows for less invasive and simple assessment of RV function, potentially promoting better understanding and management of pulmonary hypertension and other cardiovascular diseases.

Pressure-volume (PV) loop analysis is the gold standard for evaluating left/right ventricular (RV) function. Its application has become increasingly important in pulmonary arterial hypertension (PAH) because RV function critically affects PAH patients' outcome [1]. However, PV loop analysis is not used widely because it is invasive and requires dedicated catheters and expertise. Recent advances in three-dimensional (3D) echocardiography have enabled non-invasive RV volume measurements, potentially replacing conductance catheters required for RV volume measurements.
\end{abstract}

The PV loop in Figure 1 was created using data obtained from simultaneous 3D echocardiography for RV volume (Movie 1 ) and high-fidelity micromanometry for RV pressure (Figure 2 ) in a PAH patient. Using PV data along with the single-beat method, end-systolic elastance (Ees) $(1.22 \mathrm{mmHg} / \mathrm{mL})$ and Ees/[arterial elastance] (1.27), representative indices of RV systolic function and RV-pulmonary arterial coupling, respectively, were calculated. The representative index of RV relaxation, tau, was calculated as $36.3 \mathrm{~ms}$. The RV stiffness/compliance index, $\beta$, was 0.0412, calculated using Rain et al.'s formula [2].

To the best of our knowledge, the PV loop image presented in this report is the first to have been created with a simultaneously obtained RV pressure and 3D echocardiography-derived volume. This method waives the conductance catheter use, thereby significantly lessening examination time, cost, and dedicated expertise needed. With better temporal resolution of 3D echocardiography and by modifying the RV's pre/after-load, such as by Valsalva maneuver, a more detailed and less invasive RV function assessment will become possible.

Acknowledgments: We thank Ms. Azusa Nakasato, Mamiko Inoue MT, Mr. Hajime Hatanaka, and Dr. Hiroshi Ohira $\mathrm{PhD}$ for their assistance in data collection and analysis.

\section{Author contributions:}

Toshitaka Nakaya created the PV loop shown in Figure 1. Junichi Nakamura recorded the RV pressure during the catheterization using a micromanometry. Yasuyuki Chiba and Hiroyuki Iwano recorded the 3D image, analyzed the data, and created the 3D movie clip. Ichizo Tsujino conceptualized the study and reviewed the manuscript.

\section{References}

1. Vonk Noordegraaf A, Chin KM, Haddad F, et al: Pathophysiology of the right ventricle and of the pulmonary circulation in pulmonary hypertension: an update. Eur Respir J 2019;53:1801900.

2. Rain S, Handoko MP, Trip P, et al.: Right ventricular diastolic impairment in patients with pulmonary arterial hypertension. Circulation 2013;128: 2016-2025, 1-10.

Figure 1. RV pressure-volume curve of a patient with pulmonary arterial hypertension

The image was created based on a dataset of RV pressure and volume, simultaneously recorded using highfidelity micromanometry and 3D echocardiography.

$\mathrm{RV}$, right ventricular; 3D, 3-dimensional 


\section{Figure 2. RV pressure curves recorded with a high fidelity micromanometer}

RV pressure was recorded using a high-fidelity micromanometer (Mikro-Cath Pressure Cather, Millar Inc., TX, USA). The pressure catheter was connected to an AV converter (PowerLab ${ }^{\circledR}$, ADInstruments, Dunedin, New Zealand) and to a personal computer in which dedicated software (LabChart Pro ${ }^{\circledR}$, ADInstruments, Dunedin, New Zealand) was installed. This software allowed for a pressure recording with $1,000 \mathrm{~Hz}$ and for an export of the data as a csv file. Zero level was determined while the tip of the catheter was placed just below the surface of warmed water put in a cup. The RV pressure was recorded at the natural end-expiration, and the dataset of the first cardiac cycle was used for drawing the PV loop of Figure 1.

$\mathrm{RV}$, right ventricular

Supplementary material: The following supplementary material is available online (Codec ID: avc1)

\section{- Movie Clip 1: Three-dimensional movie image of the right ventricle}

RV images were recorded with a 3D echo (Aplio i800, Cannon Medical Systems, Tokyo) while the patient was asked to hold her breath at the natural end-expiration. Six consecutive beats were recorded to create a dataset of the RV volume of one cardiac cycle. The numerical data was exported afterward as a csv file and then used, along with the pressure data, to draw an RV pressure-volume loop shown in the Figure 1.

$\mathrm{RV}$, right ventricular; 3D, 3-dimensional

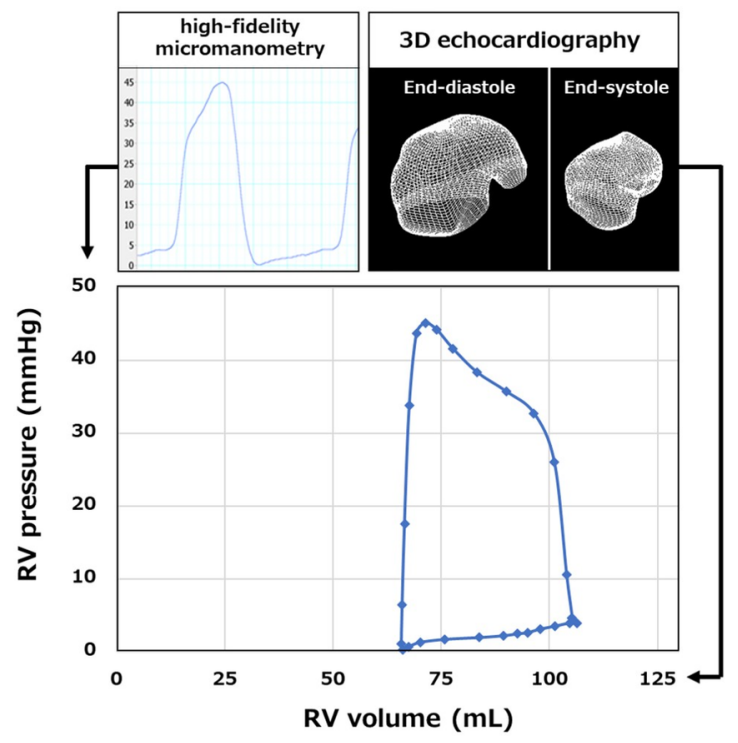




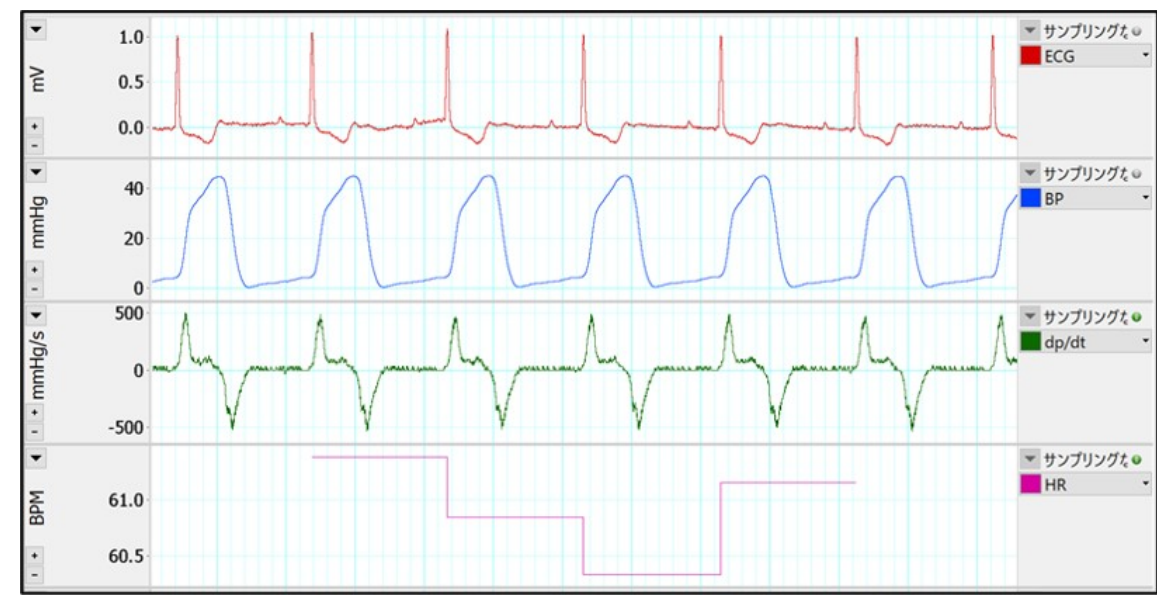

